\title{
Long-term outcome of endoscopic submucosal dissection is comparable to that of surgery for early gastric cancer: a propensity-matched analysis
}

\author{
Hye Kyung Jeon ${ }^{1}$ Gwang Ha Kim ${ }^{1}$ - Bong Eun Lee ${ }^{1}$ Do Youn Park ${ }^{2}$. \\ Geun Am Song ${ }^{1}$ Dae Hwan Kim ${ }^{3}$. Tae Yong Jeon ${ }^{3}$
}

Received: 6 February 2017/ Accepted: 3 April 2017/Published online: 10 April 2017

(C) The International Gastric Cancer Association and The Japanese Gastric Cancer Association 2017

\begin{abstract}
Background Data concerning the long-term outcomes of endoscopic submucosal dissection (ESD) versus surgery for early gastric cancer (EGC) are limited. We aimed to compare the long-term outcomes of ESD and surgery for patients with EGC.

Methods Data were reviewed from patients treated by ESD or surgery for EGC in 2005-2010. The primary outcome was overall survival (OS). Secondary outcomes were disease-specific survival (DSS), disease-free survival (DFS), recurrence-free survival (RFS), treatment-related complications, and hospital stay duration.

Results Among 617 patients, 342 underwent ESD and 275 underwent surgery. The 5-year OS rates were similar between the ESD group and the surgery group $(96.9 \%$ vs $98.1 \%, P=0.581$ ). In a propensity-score-matched analysis of 117 pairs, there were no significant differences in the OS rates $(96.5 \%$ vs $99.1 \%, P=0.125)$ and DSS rates $(100 \%$ vs $99.1 \%, P=0.317$ ) between the ESD group and the
\end{abstract}

Electronic supplementary material The online version of this article (doi:10.1007/s10120-017-0719-4) contains supplementary material, which is available to authorized users.

Gwang Ha Kim

doc0224@pusan.ac.kr

1 Department of Internal Medicine, Pusan National University School of Medicine, and Biomedical Research Institute, Pusan National University Hospital, 179, Gudeok-ro, Seo-Gu, Busan 602-739, Korea

2 Department of Pathology, Pusan National University School of Medicine, and Biomedical Research Institute, Pusan National University Hospital, Busan, Korea

3 Department of Surgery, Pusan National University School of Medicine, and Biomedical Research Institute, Pusan National University Hospital, Busan, Korea surgery group. The ESD group had a significantly lower DFS rate $(90.3 \%$ vs $98.0 \%, P=0.002)$, a significantly lower RFS rate $(95.1 \%$ vs $98.0 \%, P=0.033$ ), a significantly higher early complication rate $(6.7 \%$ vs $1.5 \%$, $P<0.001)$, a significantly lower late complication rate (0\% vs $9.1 \%, P<0.001)$, and a significantly shorter median hospital stay ( 3 days vs 10 days, $P<0.001$ ) than the surgery group.

Conclusions ESD and surgery have comparable OS rates in patients with EGC. ESD has benefits, including a lower late complication rate and shorter hospital stay. However, RFS and DFS rates might be lower after ESD than after surgery.

Keywords Early gastric cancer - Endoscopic submucosal dissection · Gastrectomy $\cdot$ Survival

\section{Introduction}

Gastric cancer is one of the leading causes of cancer-related deaths worldwide, and the second commonest cancer in Korea [1, 2]. Because of the national cancer screening program for gastric cancer initiated in 1999 [3], the proportion of patients with early gastric cancer (EGC) at diagnosis has been increasing in Korea [4]. EGC is defined as a lesion with cancer invasion confined to the mucosa or submucosa, regardless of lymph node metastasis (LNM) $[5,6]$. Surgical gastrectomy with regional lymph node dissection is a traditional standard treatment modality for EGC. The prognosis of patients with EGC after curative resection is excellent, with a 5-year overall survival (OS) rate of more than $90 \%$ [7].

Since endoscopic instruments and techniques have been developed, endoscopic resection is commonly performed 
for treatment of EGC without LNM, particularly that meeting the absolute indication criteria for endoscopic resection, such as intramucosal differentiated-type cancer $2 \mathrm{~cm}$ or smaller without ulceration [8-10]. In 2000, a largescale study proposed the expanded indication criteria for endoscopic resection on the basis of a comprehensive review to assess the risk of LNM [8]. The expanded indication criteria are (1) differentiated-type intramucosal cancer without ulceration, regardless of tumor size, (2) differentiated-type intramucosal cancer $3 \mathrm{~cm}$ or smaller, irrespective of ulceration, (3) differentiated-type minute submucosal invasive cancer $3 \mathrm{~cm}$ or smaller, and (4) undifferentiated-type intramucosal cancer $2 \mathrm{~cm}$ or smaller without ulceration [8]. With the development of endoscopic submucosal dissection (ESD), EGC meeting the expanded indication criteria can be treated successfully by ESD alone [11]. Favorable short-term and long-term clinical outcomes have been reported in many studies [12-15].

The survival rate of patients who undergo ESD for EGC meeting the absolute indication criteria is comparable to that of patients treated by surgical gastrectomy [16]. However, LNM have been reported in a small proportion of patients with EGC meeting the expanded indication criteria $[17,18]$, and controversies still exist regarding the potential risk of LNM after ESD. Therefore, it is important to compare the long-term clinical outcomes of ESD and surgery for EGC meeting the expanded indication criteria. Although the long-term outcomes of patients with EGC meeting the expanded indication criteria are reported to be similar after either ESD or surgery [19-22], there are limited data regarding the optimal treatment strategy for EGC. Therefore, we aimed to evaluate the long-term outcomes of patients who underwent ESD or surgical resection for EGC.

\section{Methods}

\section{Study design and patients}

The study included patients who underwent ESD for EGC at Pusan National University Hospital (Busan, Korea) from January 2005 to December 2010. We enrolled patients who met the following inclusion criteria: (1) older than 20 years, (2) newly diagnosed EGC, and (3) final pathologic findings of curative resection (defined later) after ESD under the absolute or expanded indication criteria. The exclusion criteria were as follows: (1) previous treatment for gastric cancer, (2) cancer of another organ, (3) follow-up duration less than 1 year, (4) additional gastrectomy after initial ESD, or (5) noncurative pathologic findings.
During the same period, patients who underwent surgical gastrectomy with lymph node dissection for EGC were enrolled for comparison with the ESD group. Among them, we excluded patients who had (1) previously been treated for gastric cancer, (2) cancer of an other organ, (3) a follow-up duration of less than 1 year, (4) deep submucosal invasive cancer (more than $500 \mu \mathrm{m}$ from the muscularis mucosae), or (5) the presence of LNM or lymphovascular invasion. This study was reviewed and approved by the Institutional Review Board of Pusan National University Hospital (H-1612-006-049).

\section{ESD and surgery}

Before treatment, all patients underwent endoscopy, endoscopic ultrasonography, and computed tomography for clinical staging. All ESD procedures were performed as described in our previous study [13]. In the surgery group, patients underwent laparoscopy-assisted or open gastrectomy with $\mathrm{D} 1+\beta$ or more lymph node dissection. The extent of lymph node dissection was based on the recommendations of the Japanese Gastric Cancer Association [10]. A total, distal, or proximal subtotal gastrectomy was conducted according to the tumor location and extent.

\section{Histopathology evaluation}

Resected specimens were fixed in a $10 \%$ formalin solution and serially sectioned to assess tumor involvement of the lateral and vertical margins; ESD specimens and surgical specimens were serially sliced at 2- and 5-mm intervals respectively. The tumor location was described as being in the upper, middle, or lower third of the stomach, and the macroscopic type was classified as elevated, flat, or depressed. Tumor size, degree of differentiation, invasion depth, presence of ulceration, and lymphovascular emboli were evaluated microscopically. Tumors were classified histopathologically according to the Japanese classification of gastric carcinoma [23]; tubular adenocarcinoma (welldifferentiated and moderately differentiated adenocarcinoma) and papillary adenocarcinoma were classified as differentiated type, and poorly differentiated adenocarcinoma, signet ring cell carcinoma, and mucinous adenocarcinoma were classified as undifferentiated type. If a cancer had histologic heterogeneity, its histologic appearance was described according to the quantitatively predominant type. Curative resection was defined if the following conditions were met: en bloc resection, tumorfree lateral and vertical margins, meeting of the expanded indication criteria, and the absence of lymphovascular invasion. 


\section{Follow-up}

In both the ESD group and the surgery group, follow-up endoscopy, abdominal computed tomography, chest radiography, and laboratory measurement of tumor markers were conducted 6 months later and annually thereafter. If patients were lost to follow-up, mortality data were obtained from the National Cancer Registry database. Follow-up data on recurrences and deaths were obtained until December 2015.

\section{Outcome data}

The primary outcome of this study was OS. Secondary outcomes included disease-specific survival (DSS), disease-free survival (DFS), recurrence-free survival (RFS), the development of metachronous gastric cancer, treatment-related complications, length of hospital stay, and 30-day readmission rates. OS was defined as the length of time from the date of ESD or surgery to the date of death from any cause, and DSS was defined as the length of time from the date of ESD or surgery to the date of death related to gastric cancer. DFS was defined as the length of time from the date of ESD or surgery to the date of the first gastric cancer recurrence, metachronous gastric cancer occurrence, or death from any cause. RFS was defined as the length of time from the date of ESD or surgery to date of the first gastric cancer recurrence, metachronous gastric cancer occurrence, or death with evidence of recurrence. Metachronous gastric cancer was defined as a new cancer that was detected at a previously uninvolved site more than 1 year after initial treatment. Early and late complications were regarded as the occurrence of adverse events within and beyond 30 days after treatment respectively.

\section{Statistical analysis}

To compare the baseline characteristics and clinicopathologic features between the ESD group and the surgery group, the chi-square test or Fisher's exact test was used for categorical variables and the Student $t$ test or the MannWhitney $U$ test was used for continuous variables. Propensity-score-matched analysis was conducted to reduce the effect of possible confounding factors and treatment-related selection bias [24, 25]. Propensity scores were determined by a logistic regression model of the covariates as follows: age, sex, comorbidities, and tumor location, size, morphology, invasion depth, and differentiation. By use of these propensity scores, patients in the ESD group were individually matched to patients in the surgery group. To assess bias reduction, we checked the balance of the matched data in terms of absolute standardized differences of covariates before and after matching. An absolute standardized difference of less than $10 \%$ suggests a substantial balance across the groups. The Kaplan-Meier method was used to estimate the long-term survival outcomes, and the log-rank test was used to analyze the statistical differences between the treatment groups. The hazard ratios (HRs) of surgery over ESD for the long-term outcomes were determined with a Cox proportional hazards model. To evaluate the independent predictors of $\mathrm{OS}$, variables with $P<0.20$ in univariate analyses were entered into a multiple regression analysis, using the Cox proportional hazards model. $P<0.05$ was considered statistically significant. Statistical calculations were performed with PASW Statistics for Windows version 18.0 (SPSS, Chicago, IL, USA).

\section{Results}

\section{Study population}

From January 2005 to December 2010, 756 patients with 777 EGCs were treated by ESD. Among them, 414 patients were excluded for the following reasons: history of gastric cancer $(n=8)$, cancer of another organ $(n=59)$, followup less than 1 year $(n=217)$, not meeting the criteria for curative resection $(n=123$; 5 with lymphovascular invasion, 11 with piecemeal resection, 26 with positive tumor margin, and 81 beyond the expanded indication criteria), and additional surgery $(n=7)$. During the same period, 873 patients underwent surgery for EGC. Among them, 598 patients were excluded for the following reasons: history of gastric cancer $(n=3)$, cancer of another organ ( $n=36$ ), follow-up less than 1 year $(n=51)$, beyond the expanded indication criteria $(n=478)$, presence of LNM $(n=24)$, and presence of lymphovascular emboli $(n=6)$. Finally, 617 patients were eligible for long-term outcome analysis (342 patients in the ESD group and 275 patients in the surgery group). Patients in the ESD group were matched individually to patients in the surgery group with use of propensity scores; consequently 117 pairs of matched patients were created (Fig. 1).

Table 1 summarizes the baseline characteristics of all the patients and propensity-score-matched patients. Overall, patients in the ESD group were older than those in the surgery group $(62.9 \pm 9.4$ years vs $57.7 \pm 10.6$ years, $P<0.001)$. Tumors were smaller $(1.4 \pm 0.9 \mathrm{~cm}$ vs $2.2 \pm 1.6 \mathrm{~cm}, P<0.001)$ and likelier to have elevated morphology and differentiated-type histologic appearance in the ESD group than in the surgery group $(P=0.017$ and $P<0.001$ respectively). In addition, the proportion of EGCs meeting the absolute indication criteria was higher in the ESD group than in the surgery group $(69.9 \%$ vs $33.5 \%$, $P<0.001)$. The proportions of male patients, preexisting 


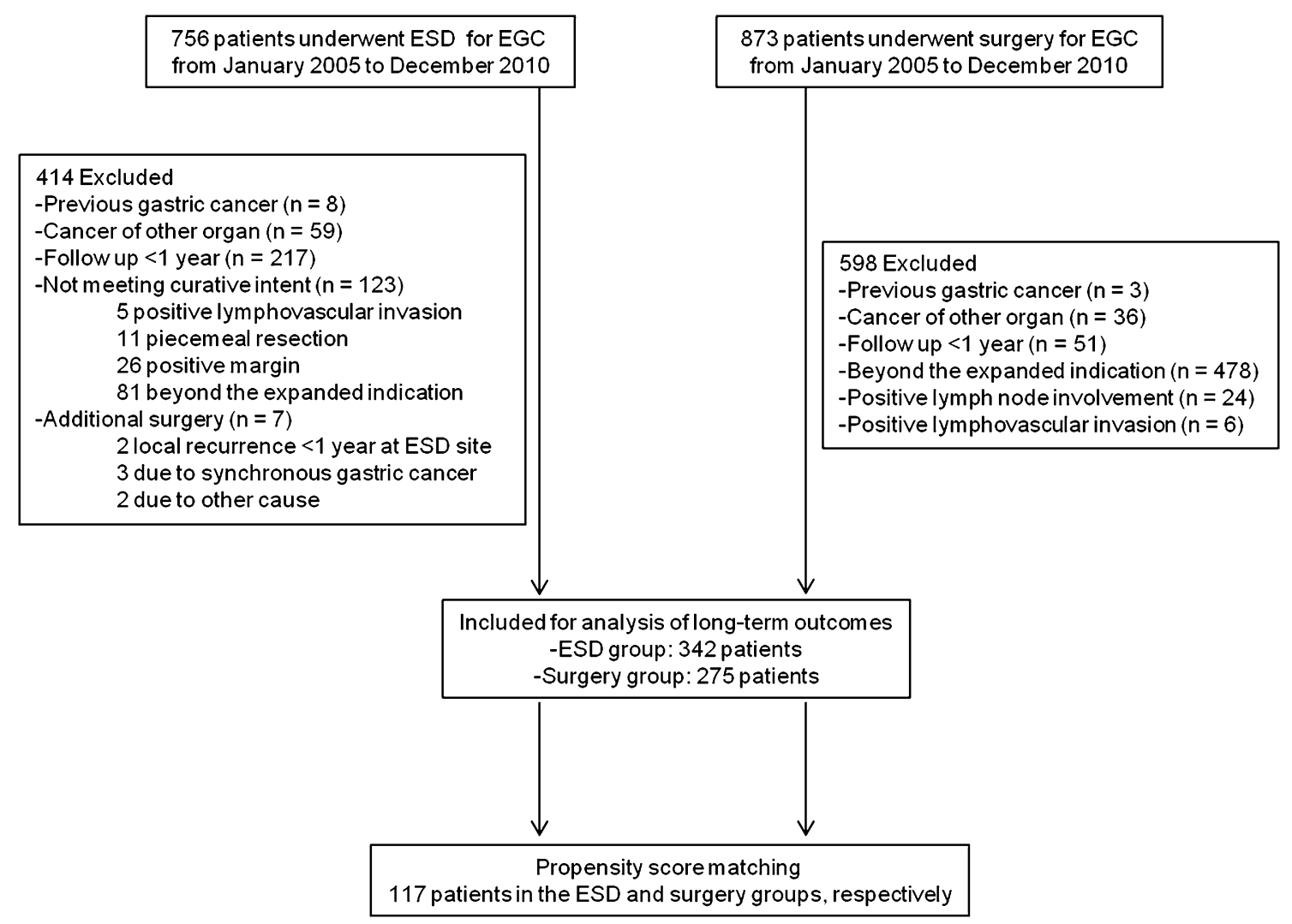

Fig. 1 Flowchart of the study population. EGC early gastric cancer, ESD endoscopic submucosal dissection

comorbidities, and the location of tumor and the invasion depth were similar between the groups. In 117 pairs of matched patients, all baseline characteristics were similar between the groups, and postmatching standardized differences for all covariates were less than $10 \%$ (Fig. 2).

\section{Long-term outcomes of ESD and surgery}

The ESD group had a median follow-up period of 58 months (range 12-122 months), and the surgery group had a median follow-up period of 58 months (range 12-130 months). Four patients died of gastric cancer in the surgery group, whereas no gastric-cancer-related death was observed in the ESD group. Metachronous gastric cancers occurred only in the ESD group (31/342, 9.1\%). The median time to metachronous gastric cancer was 36 months (range 12-98 months). Patients with metachronous gastric cancers were treated by $\operatorname{ESD}(n=29)$ and surgery $(n=1)$ or observed because of refusal of additional treatment because of old age $(n=1)$. Of these, four patients had a second metachronous gastric cancer and all lesions were also resected by ESD. Metachronous gastric cancer of the remnant stomach was not observed in the surgery group. Local recurrence was observed in one patient $(1 / 342,0.3 \%)$ in the ESD group without evidence of
LNM. The time to local recurrence was 15 months, and this patient moved to another hospital willingly for surgical treatment. In the surgery group, one patient $(1 / 275,0.3 \%)$ experienced tumor recurrence in the celiac axis lymph node 51 months after gastrectomy. Distant recurrences with liver metastasis were observed in two patients $(2 / 275,0.7 \%)$ in the surgery group at 8 and 42 months after gastrectomy respectively.

The Kaplan-Meier survival curves with log-rank test results are presented in Fig. 3, and the HRs of surgery over ESD for the long-term outcomes are presented in Table 2. There was no significant difference between the ESD group and the surgery group in 5-year OS rates (96.9\% vs $98.1 \%$, $P=0.581$, log-rank test). The DSS rate was significantly better in the ESD group than in the surgery group [100\% vs 98.5\%, $P=0.028$, log-rank test, HR 10.922, 95\% confidence interval (CI) 1.166-1447.367, $P=0.034$ ]. The RFS and DFS rates were significantly worse in the ESD group than in the surgery group (RFS rates $92.1 \%$ vs $98.7 \%$, $P<0.001$, log-rank test, HR $0.088,95 \%$ CI $0.027-0.291$, $P<0.001$; DFS rates $88.0 \%$ vs $96.9 \%, P<0.001, \log -$ rank test, HR $0.240,95 \%$ CI $0.128-0.451, P<0.001)$.

In propensity-score-matched analysis, the long-term outcomes of the ESD group were not significantly different from those of the surgery group in terms of 5-year OS rates 
Table 1 Baseline demographics of the study population

\begin{tabular}{|c|c|c|c|c|c|c|}
\hline & \multicolumn{3}{|l|}{ Before matching } & \multicolumn{3}{|l|}{ After matching } \\
\hline & $\operatorname{ESD}(n=342)$ & Surgery $(n=275)$ & $P$ & $\mathrm{ESD}(n=117)$ & Surgery $(n=117)$ & $P$ \\
\hline Age (mean $\pm \mathrm{SD}$; years) & $62.9 \pm 9.4$ & $57.7 \pm 10.6$ & $<0.001$ & $59.9 \pm 8.8$ & $59.5 \pm 9.7$ & 0.731 \\
\hline Male sex & $234(68.4 \%)$ & $176(64.0 \%)$ & 0.265 & $82(70.1 \%)$ & $81(69.2 \%)$ & $>0.999$ \\
\hline \multicolumn{7}{|l|}{ Preexisting comorbidity } \\
\hline Cardiovascular disease & $127(39.8 \%)$ & $90(36.4 \%)$ & 0.434 & $40(34.2 \%)$ & $39(33.3 \%)$ & $>0.999$ \\
\hline Respiratory disease & $10(3.1 \%)$ & $10(4.0 \%)$ & 0.648 & $4(3.4 \%)$ & $3(2.6 \%)$ & $>0.999$ \\
\hline Liver disease & $20(6.3 \%)$ & $11(4.5 \%)$ & 0.457 & $6(5.1 \%)$ & $5(4.3 \%)$ & $>0.999$ \\
\hline Renal disease & $15(4.7 \%)$ & $5(2.0 \%)$ & 0.109 & $2(1.7 \%)$ & $2(1.7 \%)$ & $>0.999$ \\
\hline Diabetes & $45(14.1 \%)$ & $37(15.0 \%)$ & 0.810 & $16(13.7 \%)$ & $18(15.4 \%)$ & 0.853 \\
\hline Tumor location & & & 0.626 & & & 0.808 \\
\hline Upper third & $19(5.6 \%)$ & $12(4.4 \%)$ & & $3(2.6 \%)$ & $2(1.7 \%)$ & \\
\hline Middle third & $103(30.1 \%)$ & $91(33.1 \%)$ & & $38(32.5 \%)$ & $35(29.9 \%)$ & \\
\hline Lower third & $220(64.3 \%)$ & $172(62.5 \%)$ & & $76(65.0 \%)$ & $80(68.4 \%)$ & \\
\hline Tumor morphology & & & 0.017 & & & 0.628 \\
\hline Elevated & $76(22.2 \%)$ & $40(14.5 \%)$ & & $22(18.8 \%)$ & $26(22.2 \%)$ & \\
\hline Flat or depressed & $266(77.8 \%)$ & $235(85.5 \%)$ & & $95(81.2 \%)$ & $91(77.8 \%)$ & \\
\hline Tumor size (mean $\pm \mathrm{SD} ; \mathrm{mm})$ & $1.4 \pm 0.9$ & $2.2 \pm 1.6$ & $<0.001$ & $1.8 \pm 1.1$ & $1.8 \pm 1.0$ & 0.903 \\
\hline Depth of invasion & & & 0.243 & & & $>0.999$ \\
\hline Mucosa & $324(94.7 \%)$ & $266(96.7 \%)$ & & $111(94.9 \%)$ & $111(94.9 \%)$ & \\
\hline Submucosa, SM1 & $18(5.3 \%)$ & $9(3.3 \%)$ & & $6(5.1 \%)$ & $6(5.1 \%)$ & \\
\hline Histologic appearance & & & $<0.001$ & & & $>0.999$ \\
\hline Differentiated & $338(98.8 \%)$ & $195(70.9 \%)$ & & $113(96.6 \%)$ & $113(96.6 \%)$ & \\
\hline Undifferentiated & $4(1.2 \%)$ & $80(29.1 \%)$ & & $4(3.4 \%)$ & $4(3.4 \%)$ & \\
\hline Indication criteria & & & $<0.001$ & & & 0.692 \\
\hline Absolute criteria & $239(69.9 \%)$ & $92(33.5 \%)$ & & $65(55.6 \%)$ & $69(59.0 \%)$ & \\
\hline Expanded criteria & $103(30.1 \%)$ & $183(66.5 \%)$ & & $52(44.4 \%)$ & $48(41.0 \%)$ & \\
\hline Follow-up duration (months) ${ }^{\mathrm{a}}$ & $58.0(36.0-66.0)$ & $58.0(49.0-72.0)$ & $0.002^{\mathrm{b}}$ & $57.0(35.5-65.5)$ & $58.0(49.0-61.0)$ & $0.367^{\mathrm{b}}$ \\
\hline Propensity score & $0.264 \pm 0.179$ & $0.607 \pm 0.296$ & $<0.001$ & $0.392 \pm 0.200$ & $0.393 \pm 0.200$ & 0.974 \\
\hline
\end{tabular}

$E S D$ endoscopic submucosal dissection, $S D$ standard deviation

a The median is given, with the interquartile range in parentheses

${ }^{\text {b }}$ Calculated by the Mann-Whitney $U$ test

(96.5\% vs $99.1 \%, P=0.125$, log-rank test) and DSS rates ( $100 \%$ vs $99.1 \%, P=0.317$, log-rank test). However, the ESD group had lower RFS and DFS rates (RFS rates 95.1\% vs $98.0 \%, P=0.033$, log-rank test, HR $0.214,95 \% \mathrm{CI}$ $0.045-1.013, P=0.052$; DFS rates $90.3 \%$ vs $98.0 \%$, $P=0.002, \log$-rank test, HR $0.179,95 \%$ CI $0.051-0.627$, $P=0.007$ )

We also compared the long-term outcomes of the ESD and surgery groups, after exclusion of EGCs that met the absolute indication criteria for endoscopic resection (Fig. S1). There was no significant difference between the ESD group and the surgery group in 5-year OS rates (97.1\% vs $98.3 \%, P=0.953$, log-rank test) and DSS rates (100\% vs $98.9 \%, P=0.289, \log$-rank test). The RFS and DFS rates were significantly lower in the ESD group than in the surgery group (RFS rates $95.5 \%$ vs $98.7 \%$,
$P<0.001$, log-rank test; DFS rates $89.2 \%$ vs $97.3 \%$, $P=0.005$, log-rank test). In propensity-score-matched analysis, the ESD and surgery groups showed comparable long-term outcomes.

In addition, we investigated predictors of OS. Old age and preexisting respiratory disease and liver disease were significantly associated with decreased OS in univariate analysis; however, we could not find any significant predictor of OS in multivariate Cox regression analysis (Table 3).

\section{Short-term outcomes}

Compared with the surgery group, the ESD group had a significantly shorter procedure time (33 $\mathrm{min}$ vs $220 \mathrm{~min}$, $P<0.001$ ) and duration of hospital stay (3 days vs 


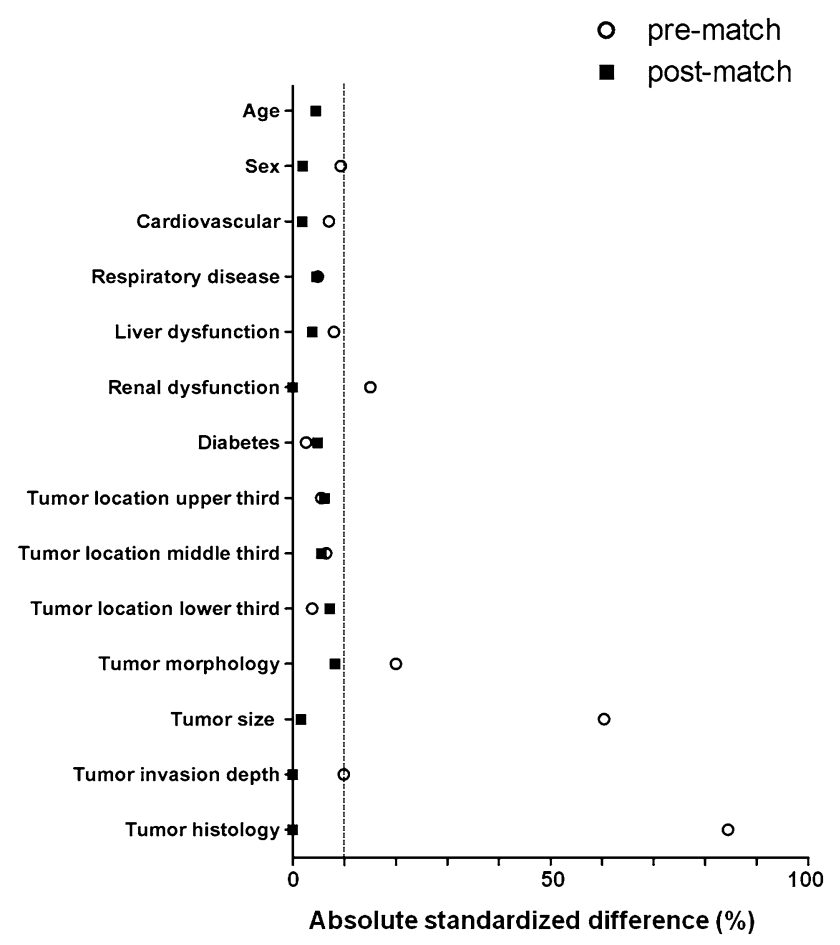

Fig. 2 Absolute standardized differences in baseline characteristics before and after propensity score matching

10 days, $P<0.001)$. There was no significant difference in the 30-day readmission rates between the ESD group and the surgery group. These results were not changed in the propensity-score-matched groups (Table 4).

\section{Treatment-related complications and hospital stay}

Early complications occurred in 23 patients from the ESD group: 22 patients with post-ESD bleeding and 1 patient with gastric perforation. All patients with post-ESD bleeding were treated successfully by endoscopic hemostasis. One patient with gastric perforation was treated by endoscopic clipping and conservative management. In the surgery group, four patients experienced early complications: three patients had intra-abdominal abscess and one patient had enterocutaneous fistula. All patients with intra-abdominal abscess were treated by conservative management; however, one patient required an additional surgical procedure. The patient with enterocutaneous fistula was treated by conservative management. The rate of early complications was significantly higher in the ESD group than in the surgery group [6.7\% (23/342) vs $1.5 \%$ (4/ 275), $P<0.001]$.

There were no late complications in the ESD group, whereas 25 patients from the surgery group experienced late complications: anastomosis site stricture in 4 patients and intestinal obstruction due to postoperative adhesion in 21 patients. All patients with anastomosis site stricture were treated by endoscopic balloon dilation. Among the 21 patients with intestinal obstruction, 10 patients were treated successfully by conservative management; however, 7 patients underwent additional surgery with adhesiolysis because of failure of conservative treatment. The ESD group had a significantly lower rate of late complications than the surgery group $[0 \%(0 / 342)$ vs $9.1 \%(25 / 275)$, $P<0.001]$.

\section{Discussion}

In the present study with a propensity-score-matched analysis of EGC that met the expanded indication criteria, ESD was comparable to surgery in terms of 5-year OS and DSS rates; however, the ESD group had 5-year RFS and DFS rates lower than those of the surgery group because of a higher occurrence of metachronous cancer. Regarding short-term outcomes, the procedure time and the hospital stay were shorter in the ESD group than in the surgery group, whereas the 30-day readmission rates were similar between groups. The ESD group had a significantly higher early complication rate than the surgery group; however, late complications occurred only in the surgery group.

ESD is a preferred treatment modality for EGC without LNM, especially in Eastern countries, including Korea and Japan. The expanded indication criteria for endoscopic resection were established on the basis of clinical observations and a large pathology review [8], and many retrospective studies have reported the benefits and short-term and long-term outcomes of ESD [12-15]. Although the risk of LNM is negligible, controversies exist in terms of the oncologic safety of ESD for EGC meeting the expanded indication criteria; therefore, additional long-term outcome studies should be widely performed to evaluate the safety and benefits of ESD over surgery so as to support the current indication criteria. In our study with a propensityscore-matched analysis, the ESD group had OS and DSS rates similar to those of the surgery group, in accordance with the findings of previous studies [16, 19-22]. During the follow-up period, recurrences at regional lymph nodes or distant recurrence were not found in the ESD group. Thus, our results support the negligible risk of LNM if the final pathologic findings meet the expanded indication criteria.

In the present study, the RFS and DFS rates were lower in the ESD group than in the surgery group, mainly due to the development of metachronous gastric cancer. The possibility of metachronous gastric cancer may be a major concern related to ESD. Despite curative resection of EGC by ESD, patients still have a potential risk of new gastric cancer at other sites in the stomach because their stomach is preserved [26, 27]. Metachronous gastric cancer can 
Fig. 3 Kaplan-Meier survival plots for the endoscopic submucosal dissection (ESD) and surgery groups for all patients (a) and for propensityscore-matched patients (b).

$P$ values were calculated by the log-rank test a

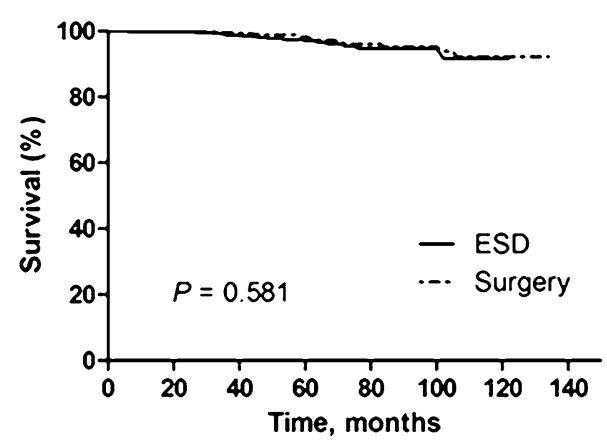

Disease specific survival

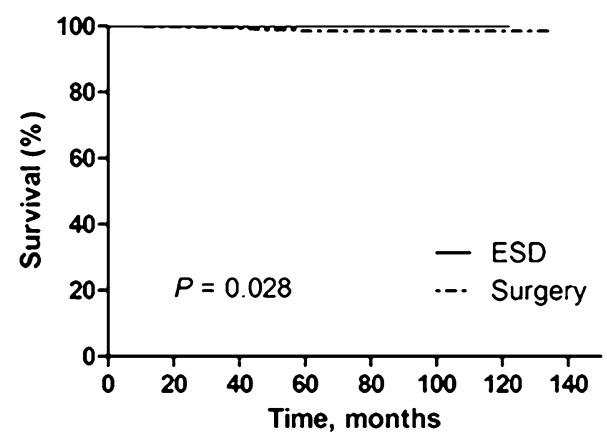

Disease free survival

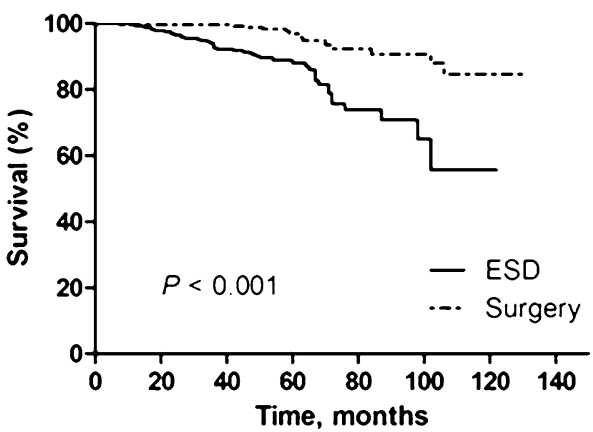

Recurrence free survival

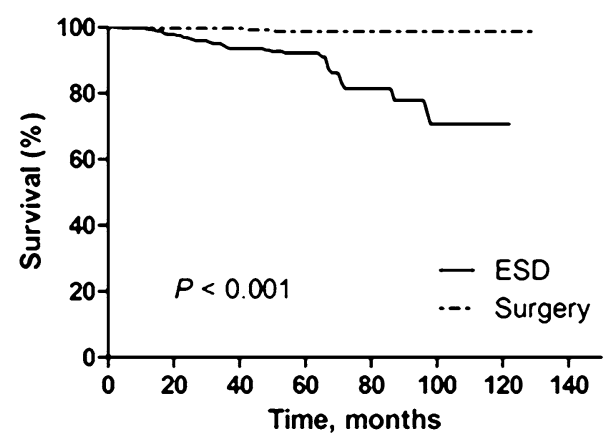

b

Overall survival

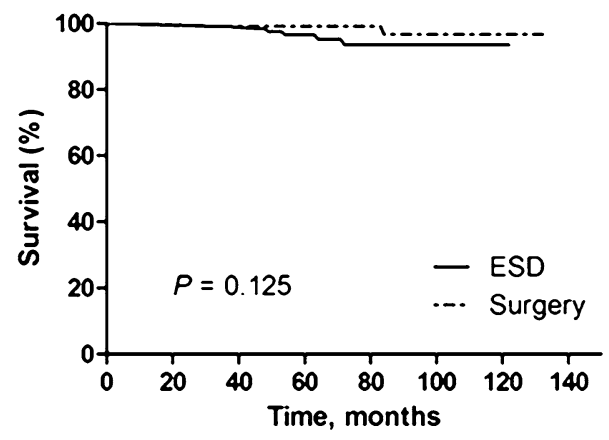

Disease specific survival

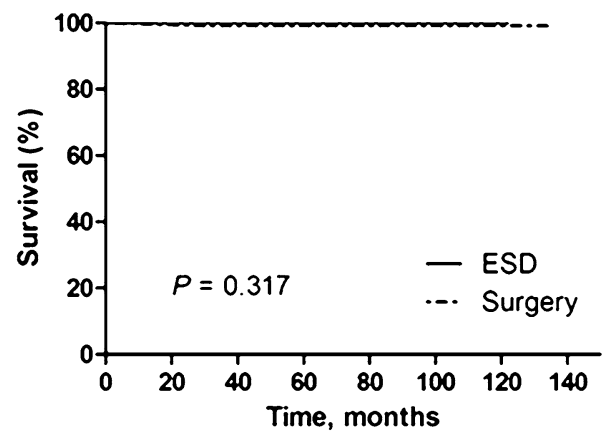

Disease free survival

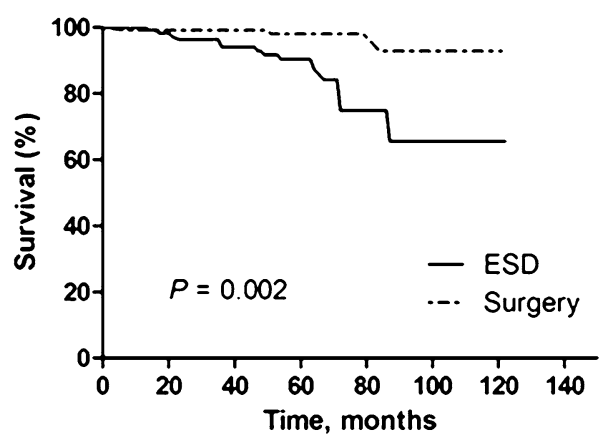

Recurrence free survival

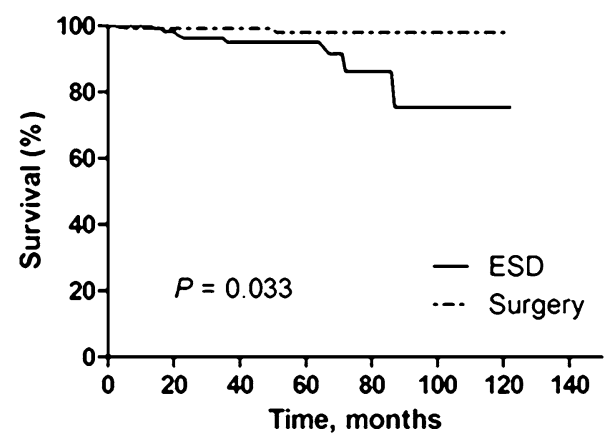

develop at a rate of $4-6.5 \%$ after endoscopic resection [13, 16, 19-21], and the incidence increases with time after ESD [26]. After partial gastrectomy, the incidence of metachronous gastric cancer is approximately $2.5-2.9 \%$ $[28,29]$; in recent studies comparing long-term survival between endoscopic resection and surgery, metachronous gastric cancer has been reported to occur in less than $1 \%$ of patients after surgery [16, 19-21]. In accordance with previous studies, our analysis showed that only patients in the ESD group developed metachronous gastric cancers, at 
Table 2 Cox proportional hazards models for long-term outcomes for all patients and for propensity-score-matched patients

\begin{tabular}{|c|c|c|c|c|}
\hline & \multicolumn{2}{|l|}{ Events } & \multirow[t]{2}{*}{ Hazard ratio $^{\mathrm{a}}$} & \multirow[t]{2}{*}{$P$ value } \\
\hline & ESD & Surgery & & \\
\hline \multicolumn{5}{|l|}{ All patients } \\
\hline Overall survival & $15(4.4 \%)$ & $12(4.4 \%)$ & $0.806(0.374-1.738)$ & 0.806 \\
\hline Disease-specific survival & 0 & $4(1.5 \%)$ & $10.922(1.166-1447.367)$ & 0.034 \\
\hline Disease-free survival & $46(13.5 \%)$ & $13(4.7 \%)$ & $0.240(0.128-0.451)$ & $<0.001$ \\
\hline Recurrence-free survival & $32(9.4 \%)$ & $3(1.1 \%)$ & $0.088(0.027-0.291)$ & $<0.001$ \\
\hline \multicolumn{5}{|c|}{ Propensity-score-matched patients } \\
\hline Overall survival & $6(5.1 \%)$ & $2(1.7 \%)$ & $0.306(0.062-1.520)$ & 0.148 \\
\hline Disease-specific survival & 0 & $1(0.9 \%)$ & $3.000(0.160-437.761)$ & 0.469 \\
\hline Disease-free survival & $14(11.9 \%)$ & $3(2.6 \%)$ & $0.179(0.051-0.627)$ & 0.007 \\
\hline Recurrence-free survival & $8(6.8 \%)$ & $2(1.7 \%)$ & $0.214(0.045-1.013)$ & 0.052 \\
\hline
\end{tabular}

$E S D$ endoscopic submucosal dissection

a The $95 \%$ confidence interval is given in parentheses a rate of $9.1 \%$. However, surveillance endoscopy at a minimum of 1-year intervals can detect metachronous cancers usually at an early stage, which can be successfully treated in most cases with repeated ESD. In the present study, most metachronous gastric cancers (29/31) were successfully treated by repeated ESD. With the exception of one patient who refused additional treatment because of old age, only one patient was treated by surgery; this patient was lost to follow-up for 5 years before the occurrence of metachronous cancer, and he underwent gastrectomy for a deep submucosal invasive cancer at diagnosis. Therefore, patients who undergo ESD warrant careful follow-up surveillance to detect metachronous cancers at an early stage.

Another important point to consider is that preserving the entire gastric function provides a better quality of life for patients treated by ESD than for those treated by surgery [30]. With regard to the good prognosis of EGC and the comparable OS rates between the ESD group and the surgery group, posttreatment quality of life cannot be overlooked. A previous multicenter study suggested that no additional treatment, even after noncurative ESD, might be an acceptable option for patients [31]; therefore, long-term quality of life should be considered seriously when a treatment method is being selected [32]. We did not assess patients' quality of life in the present study. Further study with comparison of long-term quality of life between an ESD group and a surgery group is needed to support the selection of an optional treatment strategy for patients with EGC.

In the present study, local tumor recurrence was observed in one patient in the ESD group. At the time of initial ESD, EGC was diagnosed as differentiated-type intramucosal cancer meeting the absolute indication criteria. In our previous study, the local tumor recurrence rates were higher with the expanded indication criteria than with the absolute indication criteria, and local tumor recurrence was most common in cases of noncurative resection [13]. In the present study, local tumor recurrence was relatively uncommon. The single patient underwent secondary ESD and did not experience any further recurrence.

ESD also has advantages over surgery in terms of treatment-related complications. In the present study, early complications occurred more frequently in the ESD group than in the surgery group because we included all types of bleeding, such as minor bleeding or blood clot detected by routine, second-look endoscopy within $24 \mathrm{~h}$ of the procedure. Among the patients affected, only four required an emergency endoscopy because of clinical symptoms including hematemesis or melena, and most other patients (18/22) had a small amount of oozing blood or exposed vessel identified during second-look endoscopy without clinical symptoms. All cases were successfully managed by endoscopic hemostasis. One patient with perforation was treated with endoscopic clip and conservative management, and started oral intake 5 days after ESD. On the other hand, although the rate of early complications was significantly lower in the surgery group than in the ESD group, one patient in the surgery group in whom medical treatment with antibiotics failed required additional surgical treatment for an intra-abdominal abscess. Late complications occurred only in the surgery group. Among them, 11 patients needed additional surgical treatments for intestinal obstruction. Therefore, ESD might be a safe treatment option to reduce the late complication rate and the risk of surgical-procedure-related death.

ESD required a significantly shorter hospital stay than surgery. A short hospital stay is mainly associated with early recovery to daily life as well as reduced medical costs. It is reported that patients with EGC in Korea had 
Table 3 Predictors of overall survival in multivariate Cox regression analysis from all the data

\begin{tabular}{|c|c|c|c|c|}
\hline & \multicolumn{2}{|l|}{ Univariate analysis } & \multicolumn{2}{|l|}{ Multivariate analysis } \\
\hline & Hazard ratio $^{a}$ & $P$ value & Hazard ratio $^{\mathrm{a}}$ & $P$ \\
\hline Age & $1.116(1.017-1.225)$ & 0.021 & $1.071(0.967-1.195)$ & 0.192 \\
\hline \multicolumn{5}{|l|}{ Sex } \\
\hline Male & 1.000 & & & \\
\hline Female & $0.309(0.038-2.513)$ & 0.272 & & \\
\hline \multicolumn{5}{|c|}{ Cardiovascular disease } \\
\hline No & 1.000 & & 1.000 & \\
\hline Yes & $3.369(0.805-14.112)$ & 0.096 & $1.602(0.347-8.016)$ & 0.543 \\
\hline \multicolumn{5}{|l|}{ Respiratory disease } \\
\hline No & 1.000 & & 1.000 & \\
\hline Yes & $8.116(1.632-40.366)$ & 0.011 & $4.183(0.628-21.026)$ & 0.128 \\
\hline \multicolumn{5}{|l|}{ Liver disease } \\
\hline No & 1.000 & & 1.000 & \\
\hline Yes & $1.113(0.009-8.927)$ & 0.005 & $0.780(0.006-7.525)$ & 0.867 \\
\hline \multicolumn{5}{|l|}{ Renal disease } \\
\hline No & 1.000 & & & \\
\hline Yes & $3.343(0.026-27.567)$ & 0.484 & & \\
\hline \multicolumn{5}{|l|}{ Diabetes } \\
\hline No & 1.000 & & 1.000 & \\
\hline Yes & $4.113(0.978-17.302)$ & 0.054 & $1.700(0.342-7.946)$ & 0.499 \\
\hline \multicolumn{5}{|l|}{ Tumor location } \\
\hline Upper third & 1.000 & & & \\
\hline Middle third & $0.733(0.077-97.612)$ & 0.842 & & \\
\hline Lower third & $0.316(0.033-42.005)$ & 0.506 & & \\
\hline \multicolumn{5}{|l|}{ Tumor morphology } \\
\hline Elevated & 1.000 & & & \\
\hline Flat or depressed & $0.769(0.155-3.809)$ & 0.747 & & \\
\hline Tumor size & $1.224(0.678-2.207)$ & 0.502 & & \\
\hline \multicolumn{5}{|l|}{ Depth of invasion } \\
\hline Mucosa & 1.000 & & & \\
\hline Submucosa & $1.123(0.009-9.011)$ & 0.938 & & \\
\hline \multicolumn{5}{|c|}{ Histologic appearance } \\
\hline Differentiated & 1.000 & & & \\
\hline Undifferentiated & $1.834(0.014-14.811)$ & 0.703 & & \\
\hline \multicolumn{5}{|l|}{ Treatment modality } \\
\hline ESD & 1.000 & & 1.000 & \\
\hline Surgery & $0.306(0.062-1.520)$ & 0.148 & $0.426(0.076-1.774)$ & 0.247 \\
\hline
\end{tabular}

$E S D$ endoscopic submucosal dissection

a The $95 \%$ confidence interval is given in parentheses lower medical costs with ESD, compared with surgery, mainly because of shorter hospital stay and fewer required resources [33].

In the present study, we could not identify any significant predictor of OS. A recent study reported several predictors of OS, including age, comorbidity index, performance index, sex, tumor morphology, and depth of invasion [21]. Although we also found that age and preexisting respiratory or liver diseases were significantly associated with OS in univariate analysis, there was no statistical significance in multivariate analysis. The treatment modality for EGC also did not affect OS in multivariate analysis.

This study had several limitations. First, this was a retrospective, observational study, and the baseline and clinicopathologic characteristics of both groups were different. Therefore, we performed a propensity-score-matched analysis to minimize these differences between groups. 
Table 4 Short-term outcomes of patients who underwent endoscopic submucosal dissection and surgery for early gastric cancer

\begin{tabular}{llllllll}
\hline & Before matching & & & After matching \\
\cline { 2 - 3 } & ESD $(n=342)$ & Surgery $(n=275)$ & $P$ & & ESD $(n=117)$ & Surgery $(n=117)$ & $P$ \\
\hline Median length of procedure (min) & $33(21.0-47.8)$ & $220(175.0-285.0)$ & $<0.001$ & & $37(25.0-54.0)$ & $220(175-280)$ & $<0.001$ \\
Median hospital stay (days) & $3(3-4)$ & $10(8-12)$ & $<0.001$ & $3(3-4)$ & $9(8-11)$ & $<0.001$ \\
$30-$ day readmissions & $2(0.6 \%)$ & $7(2.5 \%)$ & 0.086 & 0 & $3(2.6 \%)$ & 0.247 \\
\hline
\end{tabular}

$E S D$ endoscopic submucosal dissection

${ }^{a}$ The interquartile range is given in parentheses

Second, the numbers for minute submucosal invasive cancer and undifferentiated-type cancer were small; therefore, subgroup analysis with comparisons for each dimension of the expanded indication criteria could not be performed between groups. Third, a number of patients were excluded from this study because of a short follow-up period of less than 1 year. Fourth, because of a possible histologic discrepancy between pretreatment and posttreatment diagnoses, our results have limitations to describe the long-term outcomes of EGC in which pretreatment diagnosis meets the expanded indication criteria, because our results were obtained on the basis of the final pathologic findings for the resected specimen. Further prospective multicenter studies are needed to confirm our results.

In conclusion, ESD might be a good treatment choice for EGC when the expanded indication criteria for endoscopic resection are met. The OS rate of ESD patients is comparable to that of those who undergo surgery. The benefits of ESD include low incidence of late complications and a short hospital stay. However, the risk of metachronous gastric cancer is higher in ESD patients than in surgery patients; therefore, careful regular surveillance endoscopy is essential to detect metachronous cancer at an early stage.

Acknowledgements This study was supported by a grant from the National R\&D Program for Cancer Control, Ministry for Health, Welfare and Family Affairs, Republic of Korea (0920050), and by the Medical Research Center Program through the National Research Foundation of Korea grant funded by the Korean government (NRF2015R1A5A2009656).

\section{Compliance with ethical standards}

Conflict of interest The authors declare that they have no competing interests.

Human rights statement All procedures followed were in accordance with the ethical standards of the responsible committee on human experimentation (institutional and national) and with the Declaration of Helsinki of 1964 and later versions.

Informed consent Informed consent or a substitute for it was obtained from all patients before they were included in the study.

\section{References}

1. Jung KW, Won YJ, Kong HJ, Oh CM, Cho H, Lee DH, et al. Cancer statistics in Korea: incidence, mortality, survival, and prevalence in 2012. Cancer Res Treat. 2015;47:127-41.

2. Jemal A, Bray F, Center MM, Ferlay J, Ward E, Forman D. Global cancer statistics. CA Cancer J Clin. 2011;61:69-90.

3. Choi IJ. Gastric cancer screening and diagnosis. Korean J Gastroenterol. 2009;54:67-76.

4. Nam SY, Choi IJ, Park KW, Kim CG, Lee JY, Kook MC, et al. Effect of repeated endoscopic screening on the incidence and treatment of gastric cancer in health screenees. Eur J Gastroenterol Hepatol. 2009;21:855-60.

5. Carter KJ, Schaffer HA, Ritchie WP Jr. Early gastric cancer. Ann Surg. 1984;199:604-9.

6. Everett SM, Axon AT. Early gastric cancer in Europe. Gut. 1997:41:142-50.

7. Itoh H, Oohata Y, Nakamura K, Nagata T, Mibu R, Nakayama F. Complete ten-year postgastrectomy follow-up of early gastric cancer. Am J Surg. 1989;158:14-6.

8. Gotoda T, Yanagisawa A, Sasako M, Ono H, Nakanishi Y, Shimoda $\mathrm{T}$, et al. Incidence of lymph node metastasis from early gastric cancer: estimation with a large number of cases at two large centers. Gastric Cancer. 2000;3:219-25.

9. Soetikno R, Kaltenbach T, Yeh R, Gotoda T. Endoscopic mucosal resection for early cancers of the upper gastrointestinal tract. J Clin Oncol. 2005;23:4490-8.

10. Japanese Gastric Cancer Association. Japanese gastric cancer treatment guidelines 2010 (ver. 3). Gastric Cancer. 2011;14:113-23.

11. Oka S, Tanaka S, Kaneko I, Mouri R, Hirata M, Kawamura T, et al. Advantage of endoscopic submucosal dissection compared with EMR for early gastric cancer. Gastrointest Endosc. 2006;64:877-83.

12. Isomoto H, Shikuwa S, Yamaguchi N, Fukuda E, Ikeda K, Nishiyama H, et al. Endoscopic submucosal dissection for early gastric cancer: a large-scale feasibility study. Gut. 2009;58:331-6.

13. Choi MK, Kim GH, Park DY, Song GA, Kim DU, Ryu DY, et al. Long-term outcomes of endoscopic submucosal dissection for early gastric cancer: a single-center experience. Surg Endosc. 2013;27:4250-8.

14. Ahn JY, Jung HY. Long-term outcome of extended endoscopic submucosal dissection for early gastric cancer with differentiated histology. Clin Endosc. 2013;46:463-6.

15. Gotoda T, Iwasaki M, Kusano C, Seewald S, Oda I. Endoscopic resection of early gastric cancer treated by guideline and expanded National Cancer Centre criteria. $\mathrm{Br}$ J Surg. 2010;97:868-71.

16. Choi IJ, Lee JH, Kim YI, Kim CG, Cho SJ, Lee JY, et al. Longterm outcome comparison of endoscopic resection and surgery in 
early gastric cancer meeting the absolute indication for endoscopic resection. Gastrointest Endosc. 2015;81:333-41.

17. Kang HJ, Kim DH, Jeon TY, Lee SH, Shin N, Chae SH, et al. Lymph node metastasis from intestinal-type early gastric cancer: experience in a single institution and reassessment of the extended criteria for endoscopic submucosal dissection. Gastrointest Endosc. 2010;72:508-15.

18. Chung JW, Jung HY, Choi KD, Song HJ, Lee GH, Jang SJ, et al. Extended indication of endoscopic resection for mucosal early gastric cancer: analysis of a single center experience. J Gastroenterol Hepatol. 2011;26:884-7.

19. Kim YI, Kim YW, Choi IJ, Kim CG, Lee JY, Cho SJ, et al. Longterm survival after endoscopic resection versus surgery in early gastric cancers. Endoscopy. 2015;47:293-301.

20. Cho JH, Cha SW, Kim HG, Lee TH, Cho JY, Ko WJ, et al. Longterm outcomes of endoscopic submucosal dissection for early gastric cancer: a comparison study to surgery using propensity score-matched analysis. Surg Endosc. 2016;30:3762-73.

21. Pyo JH, Lee H, Min BH, Lee JH, Choi MG, Lee JH, et al. Longterm outcome of endoscopic resection vs. surgery for early gastric cancer: a non-inferiority-matched cohort study. Am J Gastroenterol. 2016;111:240-9.

22. Fukunaga S, Nagami Y, Shiba M, Ominami M, Tanigawa T, Yamagami $\mathrm{H}$, et al. Long-term prognosis of expanded-indication differentiated-type early gastric cancer treated with endoscopic submucosal dissection or surgery using propensity score analysis. Gastrointest Endosc. 2017;85:143-52.

23. Japanese Gastric Cancer Association. Japanese classification of gastric carcinoma: 3rd English edition. Gastric Cancer. 2011;14:101-12.

24. D'Agostino RB. Propensity score methods for bias reduction in the comparison of a treatment to a non-randomized control group. Stat Med. 1998;17:2265-81.

25. Gotoda T, Hatta W. Are randomized control studies needed to evaluate the efficacy of treatment techniques that are clearly minimally invasive and already widely used? Gastrointest Endosc. 2017;85:153-4.

26. Abe S, Oda I, Suzuki H, Nonaka S, Yoshinaga S, Nakajima T, et al. Long-term surveillance and treatment outcomes of metachronous gastric cancer occurring after curative endoscopic submucosal dissection. Endoscopy. 2015;47:1113-8.

27. Kato M, Nishida T, Yamamoto K, Hayashi S, Kitamura S, Yabuta $\mathrm{T}$, et al. Scheduled endoscopic surveillance controls secondary cancer after curative endoscopic resection for early gastric cancer: a multicentre retrospective cohort study by Osaka University ESD study group. Gut. 2013;62:1425-32.

28. Hosokawa O, Kaizaki Y, Watanabe K, Hattori M, Douden K, Hayashi $\mathrm{H}$, et al. Endoscopic surveillance for gastric remnant cancer after early cancer surgery. Endoscopy. 2002;34:469-73.

29. Nozaki I, Hato S, Kobatake T, Ohta K, Kubo Y, Nishimura R, et al. Incidence of metachronous gastric cancer in the remnant stomach after synchronous multiple cancer surgery. Gastric Cancer. 2014;17:61-6.

30. Choi JH, Kim ES, Lee YJ, Cho KB, Park KS, Jang BK, et al. Comparison of quality of life and worry of cancer recurrence between endoscopic and surgical treatment for early gastric cancer. Gastrointest Endosc. 2015;82:299-307.

31. Hatta W, Gotoda T, Oyama T, Kawata N, Takahashi A, Yoshifuku $\mathrm{Y}$, et al. Is radical surgery necessary in all patients who do not meet the curative criteria for endoscopic submucosal dissection in early gastric cancer? A multi-center retrospective study in Japan. J Gastroenterol. 2017;52:175-84.

32. Gotoda T, Yang HK. The desired balance between treatment and curability in treatment planning for early gastric cancer. Gastrointest Endosc. 2015;82:308-10.

33. Kim Y, Kim YW, Choi IJ, Cho JY, Kim JH, Kwon JW, et al. Cost comparison between surgical treatments and endoscopic submucosal dissection in patients with early gastric cancer in Korea. Gut Liver. 2015;9:174-80. 